\title{
Oscillatory behavior of a third-order neutral dynamic equation with distributed delays
}

\author{
Said R. Grace ${ }^{1}$, John R. Graef ${ }^{\bowtie 2}$ and Ercan Tunç ${ }^{3}$ \\ ${ }^{1}$ Department of Engineering Mathematics, Faculty of Engineering, Cairo University \\ Orman, Giza 12221, Egypt \\ ${ }^{2}$ Department of Mathematics, University of Tennessee at Chattanooga, Chattanooga, TN 37403, USA \\ ${ }^{3}$ Department of Mathematics, Faculty of Arts and Sciences, Gaziosmanpasa University \\ 60240, Tokat, Turkey
}

Appeared 11 August 2016

Communicated by Tibor Krisztin

Abstract. The authors present some new oscillation criteria for the third-order neutral dynamic equation with distributed delays

$$
\left[r(t)\left(\left[x(t)+\int_{a}^{b} p(t, \eta) x[\tau(t, \eta)] \Delta \eta\right]^{\Delta \Delta}\right)^{\alpha}\right]^{\Delta}+\int_{c}^{d} q(t, \xi) f(x[\phi(t, \xi)]) \Delta \xi=0
$$

on a time scale $\mathbb{T}$, where $\alpha$ is the quotient of odd positive integers. Using a Riccati type transformation and a comparison technique, they establish some new sufficient conditions to ensure that a solution $x$ of this equation either oscillates or satisfies $\lim _{t \rightarrow \infty} x(t)=0$.

Keywords: oscillation, time scales, third-order neutral dynamic equation, asymptotic behavior, distributed delays.

2010 Mathematics Subject Classification: 34K11, 34C15, 34K12, 34K40, 34N05.

\section{Introduction}

We are interested in the oscillatory behavior of third-order neutral dynamic equations with continuously distributed delays of the form

$$
\left[r(t)\left(\left[x(t)+\int_{a}^{b} p(t, \eta) x[\tau(t, \eta)] \Delta \eta\right]^{\Delta \Delta}\right)^{\alpha}\right]^{\Delta}+\int_{c}^{d} q(t, \xi) f(x[\phi(t, \xi)]) \Delta \xi=0
$$

on an arbitrary time scale $\mathbb{T}$, where $\alpha$ is a quotient of odd positive integers.

Dynamic equations on time scales have received a great deal of attention in the last twenty years. We refer the reader to the monographs of Bohner and Peterson $[2,3]$ and the survey paper of Agarwal et al. [1] for background and details on the time scale calculus. The oscillatory

\footnotetext{
${ }^{\bowtie}$ Corresponding author. Email: John-Graef@utc.edu
} 
and asymptotic behavior of solutions of dynamic equations is an active and important area of research, and we refer the reader to the papers $[7-11,15,16]$ as examples of recent results on this topic. Oscillation results for dynamic equations with distributed delays are far less prevalent in the literature. For example, Candan [4] (also see Candan [5] and Chen and Liu [6]) studied the second-order neutral dynamic equation with distributed deviating arguments

$$
\left(r(t)\left((y(t)+p(t) y(\tau(t)))^{\Delta}\right)^{\gamma}\right)^{\Delta}+\int_{c}^{d} f(t, y[\theta(t, \xi)]) \Delta \xi=0,
$$

where $\gamma>0$ is a ratio of odd positive integers and $r(t)$ and $p(t)$ are positive rd-continuous functions defined on a time scale $\mathbb{T}$, and he obtained some new sufficient conditions to ensure the oscillation of all solutions.

To the best of our knowledge, there appears to be very little known about the oscillatory and asymptotic behavior of solutions of third order neutral dynamic equations with distributed delays. Şenel and Utku [16] (also see $[13,17]$ ) have obtained some such results for equation (1.1). Our purpose here is to establish some new oscillation criteria for this equation different from those in $[13,16,17]$ (see Remark 3.3 below) and to contribute to the growing body of research on third order neutral delay dynamic equations in general and those with distributed delays in particular.

We will make use of the following conditions where $C_{r d}$ denotes the class of rd-continuous functions.

(H1) $r \in C_{r d}\left(\left[t_{0}, \infty\right)_{\mathbb{T}}, \mathbb{R}^{+}\right)$and

$$
\int_{t_{0}}^{\infty}\left(\frac{1}{r(t)}\right)^{1 / \alpha} \Delta t=\infty ;
$$

(H2) $q(t, \xi) \in C_{r d}\left(\left[t_{0}, \infty\right)_{\mathbb{T}} \times[c, d], \mathbb{R}^{+}\right), p(t, \eta) \in C_{r d}\left(\left[t_{0}, \infty\right)_{\mathbb{T}} \times[a, b], \mathbb{R}\right)$, and $0 \leq p(t) \equiv$ $\int_{a}^{b} p(t, \eta) \Delta \eta \leq P<1$

(H3) $\tau(t, \eta) \in C_{r d}\left(\left[t_{0}, \infty\right)_{\mathbb{T}} \times[a, b], \mathbb{T}\right)$ is nondecreasing in $\eta$,

$$
\tau(t, \eta) \leq t, \quad \text { and } \quad \lim _{t \rightarrow \infty} \min _{\eta \in[a, b]} \tau(t, \eta)=\infty
$$

(H4) $\phi(t, \xi) \in C_{r d}\left(\left[t_{0}, \infty\right)_{\mathbb{T}} \times[c, d], \mathbb{T}\right)$ is nondecreasing in $\xi$,

$$
\phi(t, \xi) \leq t, \quad \text { and } \quad \lim _{t \rightarrow \infty} \min _{\xi \in[c, d]} \phi(t, \xi)=\infty ;
$$

(H5) $f \in C(\mathbb{R}, \mathbb{R})$ satisfies $u f(u)>0$ for $x \neq 0$ and there exist constants $k>0$ and $\beta \leq \alpha$, with $\beta$ the ratio of odd positive integers, such that $f(u) / u^{\beta} \geq k$ for $u \neq 0$.

Defining the function

$$
z(t)=x(t)+\int_{a}^{b} p(t, \eta) x[\tau(t, \eta)] \Delta \eta
$$

equation (1.1) can be written as

$$
\left[r(t)\left(z^{\Delta \Delta}(t)\right)^{\alpha}\right]^{\Delta}+\int_{c}^{d} q(t, \xi) f(x[\phi(t, \xi)]) \Delta \xi=0 .
$$

A solution $x(t)$ of (1.1) is said to be oscillatory if it is neither eventually positive nor eventually negative, and it is non-oscillatory otherwise. 


\section{Some preliminary lemmas}

In order to prove our main results, we will make use of a special case of Keller's chain rule (see Bohner and Peterson [2, Theorem 1.90]), namely,

$$
\left(z^{\alpha}(t)\right)^{\Delta}=\alpha\left\{\int_{0}^{1}\left[h z^{\sigma}(t)+(1-h) z(t)\right]^{\alpha-1} d h\right\} z^{\Delta}(t)
$$

where $z(t)$ is a delta differentiable function.

The following lemma is rather standard when studying the oscillatory behavior of solutions of third order equations; its proof can easily be modeled, for example, after the one of Hassan and Grace [13, Lemma 2.1], Şenel and Utku [16, Lemma 2.1], or many other authors.

Lemma 2.1. Assume that conditions (H1)-(H5) hold and let $x(t)$ be a positive solution of (1.1) with $z(t)$ defined as in (1.3). Then for sufficiently large $t$, either

$$
\begin{aligned}
& \text { (I) } z(t)>0, z^{\Delta}(t)>0, z^{\Delta \Delta}(t)>0 \text {, and }\left[r(t)\left(z^{\Delta \Delta}(t)\right)^{\alpha}\right]^{\Delta}<0 \text {, or } \\
& \text { (II) } z(t)>0, z^{\Delta}(t)<0, z^{\Delta \Delta}(t)>0 \text { and }\left[r(t)\left(z^{\Delta \Delta}(t)\right)^{\alpha}\right]^{\Delta}<0 \text {. }
\end{aligned}
$$

Variations of the following lemma can be found, for example, in Hassan and Grace [13, Lemma 2.10] and Şenel and Utku [16, Lemma 2.2], and their proofs can easily be adopted to our situation.

Lemma 2.2. Assume that conditions (H1)-(H5) hold and let $x(t)$ be an eventually positive solution of (1.1) with $z(t)$ satisfying property (II). If

$$
\int_{t_{0}}^{\infty} \int_{v}^{\infty}\left[\frac{1}{r(u)} \int_{u}^{\infty} q(s) \Delta s\right]^{\frac{1}{\alpha}} \Delta u \Delta v=\infty,
$$

where $q(t)=\int_{c}^{d} q(t, \xi) \Delta \xi$, then $\lim _{t \rightarrow \infty} x(t)=0$.

Remark 2.3. Clearly, analogous results hold for eventually negative solutions of equation (1.1). In [16], the authors assumed that $\alpha=\beta$, but that is not needed for the above two lemmas.

In the following two lemmas, we consider the second order dynamic equation

$$
\left(r(t)\left(x^{\Delta}(t)\right)^{\alpha}\right)^{\Delta}=c Q(t) x^{\beta}(h(t))
$$

where $c$ is a positive constant, and $\alpha, \beta$, and $r$ are as in (1.1), Q:T $\rightarrow \mathbb{R}^{+}$and $h: \mathbb{T} \rightarrow \mathbb{T}$ are rd-continuous functions, $h^{\Delta}(t) \geq 0, h(t)<t$, and $\lim _{t \rightarrow \infty} h(t)=\infty$.

Lemma 2.4. Let condition (H1) hold. If

$$
\underset{t \rightarrow \infty}{\limsup } \int_{h(t)}^{t} Q(s)\left(\int_{h(s)}^{h(t)} r^{-1 / \alpha}(v) \Delta v\right)^{\beta} \Delta s> \begin{cases}1 / c, & \text { if } \beta=\alpha, \\ 0, & \text { if } \beta<\alpha,\end{cases}
$$

then all bounded solutions of equation (2.2) are oscillatory. 
Proof. Let $x(t)$ be a bounded nonoscillatory solution of equation (2.2), say $x(t)>0$ and $x(h(t))>0$ for $t \in\left[t_{1}, \infty\right)_{\mathbb{T}}$ for some $t_{1} \geq t_{0}$. Then, there exists $t_{2} \in\left[t_{1}, \infty\right)_{\mathbb{T}}$ such that

$$
x(t)>0, \quad x^{\Delta}(t)<0, \quad \text { and } \quad\left(r(t)\left(x^{\Delta}(t)\right)^{\alpha}\right)^{\Delta} \geq 0 \quad \text { for } t \in\left[t_{2}, \infty\right)_{\mathbb{T}} .
$$

Now, for $v \geq u \geq t_{2}$, we have

$$
\begin{aligned}
x(u)-x(v) & =-\int_{u}^{v} x^{\Delta}(\tau) \Delta \tau \\
& =-\int_{u}^{v} r^{-1 / \alpha}(\tau)\left(r(\tau)\left(x^{\Delta}(\tau)\right)^{\alpha}\right)^{1 / \alpha} \Delta \tau \\
& \geq\left(\int_{u}^{v} r^{-1 / \alpha}(\tau) \Delta \tau\right)\left(-r(v)\left(x^{\Delta}(v)\right)^{\alpha}\right)^{1 / \alpha} .
\end{aligned}
$$

For $t \geq s \geq t_{2}$, setting $u=h(s)$ and $v=h(t)$ in (2.5), we obtain

$$
x(h(s)) \geq\left(\int_{h(s)}^{h(t)} r^{-1 / \alpha}(\tau) \Delta \tau\right)\left(-r(h(t))\left(x^{\Delta}(h(t))\right)^{\alpha}\right)^{1 / \alpha} .
$$

Integrating (2.2) from $h(t) \geq t_{2}$ to $t$, we have

$$
\begin{aligned}
-r(h(t))\left(x^{\Delta}(h(t))\right)^{\alpha} & \geq r(t)\left(x^{\Delta}(t)\right)^{\alpha}-r(h(t))\left(x^{\Delta}(h(t))\right)^{\alpha} \\
& =\int_{h(t)}^{t} c Q(s) x^{\beta}(h(s)) \Delta s .
\end{aligned}
$$

Using (2.6) in (2.7) gives

$$
-r(h(t))\left(x^{\Delta}(h(t))\right)^{\alpha} \geq c \int_{h(t)}^{t} Q(s)\left(\int_{h(s)}^{h(t)} r^{-1 / \alpha}(\tau) \Delta \tau\right)^{\beta} \Delta s\left(-r(h(t))\left(x^{\Delta}(h(t))\right)^{\alpha}\right)^{\beta / \alpha},
$$

i.e.,

$$
\frac{1}{c}\left(-r(h(t))\left(x^{\Delta}(h(t))\right)^{\alpha}\right)^{1-\frac{\beta}{\alpha}} \geq \int_{h(t)}^{t} Q(s)\left(\int_{h(s)}^{h(t)} r^{-1 / \alpha}(\tau) \Delta \tau\right)^{\beta} \Delta s .
$$

Taking the limsup as $t \rightarrow \infty$ of both sides of the above inequality, we see that if $\alpha=\beta$, the contradiction is clear.

If $\beta<\alpha$, the left hand side of (2.8) is positive and must decrease to zero as $t$ increases in order to prevent a contradiction to the positivity of $x(t)$. This contradicts (2.3) and completes the proof of the lemma.

Lemma 2.5. Let (H1) hold. If

$$
\limsup _{t \rightarrow \infty} \int_{h(t)}^{t}\left(\frac{1}{r(u)} \int_{u}^{t} Q(s) \Delta s\right)^{1 / \alpha} \Delta u> \begin{cases}c^{-1 / \alpha}, & \text { if } \beta=\alpha, \\ 0, & \text { if } \beta<\alpha,\end{cases}
$$

then all bounded solutions of equation (2.2) are oscillatory.

Proof. Let $x(t)$ be a bounded nonoscillatory solution of equation (2.2), say $x(t)>0$ and $x(h(t))>0$ for $t \in\left[t_{1}, \infty\right)_{\mathbb{T}}$ for some $t_{1} \geq t_{0}$. As in the proof of Lemma 2.4, we obtain (2.4). Integrating equation (2.2) from $u$ to $t \geq u \geq t_{2}$, we have

$$
r(t)\left(x^{\Delta}(t)\right)^{\alpha}-r(u)\left(x^{\Delta}(u)\right)^{\alpha}=\int_{u}^{t} c Q(s) x^{\beta}(h(s)) \Delta s,
$$


or

$$
-x^{\Delta}(u) \geq c^{1 / \alpha}\left(\frac{1}{r(u)} \int_{u}^{t} Q(s) \Delta s\right)^{1 / \alpha} x^{\beta / \alpha}(h(t))
$$

Integrating this inequality from $h(t)$ to $t$, we obtain

$$
x(h(t)) \geq c^{1 / \alpha}\left[\int_{h(t)}^{t}\left(\frac{1}{r(u)} \int_{u}^{t} Q(s) \Delta s\right)^{1 / \alpha} \Delta u\right] x^{\beta / \alpha}(h(t)),
$$

or

$$
c^{-1 / \alpha} x^{1-\beta / \alpha}(h(t)) \geq \int_{h(t)}^{t}\left(\frac{1}{r(u)} \int_{u}^{t} Q(s) \Delta s\right)^{1 / \alpha} \Delta u .
$$

The remainder of the proof is similar to that of Lemma 2.4 and hence is omitted.

Remark 2.6. It follows from Lemmas 2.4 and 2.5 that equation (2.2) has no solution $x(t)$ satisfying $x(t) x^{\Delta}(t)<0$ for large $t$.

Lemma 2.7. Let conditions (H1)-(H5) hold and assume that $x$ is an eventually positive solution of equation (1.1) with the corresponding $z$ satisfying Case (II) of Lemma 2.1. Then there exists $\theta>1$ with $P \theta<1$ such that either

$$
x(t) \geq\left(\frac{1-P \theta}{\theta}\right) z(t)
$$

for large $t$, or $\lim _{t \rightarrow \infty} x(t)=0$.

Proof. Choose $t_{1} \in \mathbb{T}$ so that $x(t)>0$ and $x[\tau(t, \eta)]>0$ for $t \in\left[t_{1}, \infty\right)_{\mathbb{T}}$ for some $t_{1} \geq t_{0}$. Since $z(t)$ satisfies Case (II) of Lemma 2.1, there exists a constant $\kappa$ such that

$$
\lim _{t \rightarrow \infty} z(t)=\kappa<\infty
$$

(i) If $\kappa>0$, then there exists $t_{2} \geq t_{1}$ and $\theta>1$ with $\theta P<1$ such that

$$
\kappa<z(t)<\kappa \theta
$$

for $t \in\left[t_{2}, \infty\right)_{\mathbb{T}}$. Now,

$$
x(t)=z(t)-\int_{a}^{b} p(t, \eta) x[\tau(t, \eta)] \Delta \eta
$$

and so

$$
\begin{aligned}
x(t) \geq \kappa-\kappa \theta P & =\left(\frac{1-P \theta}{\theta}\right) \kappa \theta \\
& \geq\left(\frac{1-P \theta}{\theta}\right) z(t) \quad \text { for } t \in\left[t_{2}, \infty\right)_{\mathbb{T}} .
\end{aligned}
$$

(ii) If $\kappa=0$, then $\lim _{t \rightarrow \infty} z(t)=0$. Since $0<x(t) \leq z(t)$ on $\left[t_{2}, \infty\right)_{\mathbb{T}}, \lim _{t \rightarrow \infty} x(t)=0$. This completes the proof of the lemma. 


\section{Main results}

In this section, we establish some new criteria for the oscillation of equation (1.1). It will be convenient to employ the following notations.

Let

$$
q(t)=\int_{c}^{d} q(t, \xi) \Delta \xi, \quad \phi_{1}(t)=\phi(t, c), \quad \text { and } \quad \phi_{2}(t)=\phi(t, d),
$$

where $\phi(t, \xi)$ is given in (H4) and $\lambda>1$ is a constant such that $\lambda \phi_{2}(t) \leq t$ for $t \in\left[t_{0}, \infty\right)_{\mathbb{T}}$. In addition, let $c_{1}=k(1-P)^{\beta}$ and for any $t_{1} \in\left[t_{0}, \infty\right)_{\mathbb{T}}$, let

$$
\begin{aligned}
R\left(t, t_{1}\right) & =\int_{t_{1}}^{t} r^{-1 / \alpha}(s) \Delta s, \\
u(t) & =\left(\int_{t_{1}}^{t} R\left(s, t_{1}\right) \Delta s\right)^{-1},
\end{aligned}
$$

and

$$
\eta(t)=\left[\theta_{1} t+\theta_{2} \int_{t_{1}}^{t} \int_{t_{1}}^{u} r^{-1 / \alpha}(s) \Delta s \Delta u\right]^{-1}
$$

for all $t \in\left[t_{1}, \infty\right)_{\mathbb{T}}$ and any positive constants $\theta_{1}$ and $\theta_{2}$.

Theorem 3.1. Let conditions (H1)-(H5) hold and assume there exists a positive, nondecreasing, deltadifferentiable function $g(t)$ such that, for any positive constants $c_{2}$ and $c_{3}$ and $T>T_{1} \geq t_{0}$, we have

$$
\limsup _{t \rightarrow \infty} \int_{T}^{t}\left[c_{1} c_{2} \eta^{\beta}(s) g(s) q(s)-\frac{(\alpha / \beta)^{\alpha}}{(\alpha+1)^{\alpha+1}} \frac{\left(\left(g^{\Delta}(s)\right)_{+}\right)^{\alpha+1}}{\left(\gamma(s) g(s) R\left(s, T_{1}\right)\right)^{\alpha}}\right] \Delta s=\infty
$$

for $t \in[T, \infty)_{\mathbb{T}}$, where $\left(g^{\Delta}(t)\right)_{+}=\max \left\{0, g^{\Delta}(t)\right\}$, and

$$
\gamma(t)= \begin{cases}1, & \text { if } \beta=\alpha, \\ c_{3}\left(\eta^{\sigma}(t)\right)^{\frac{\alpha-\beta}{\alpha},}, & \text { if } \beta<\alpha .\end{cases}
$$

If condition (2.3) or (2.9) holds with

$$
Q(t)=\phi_{2}^{\beta}(t) q(t), \quad c=k\left(\frac{(1-P \theta)(\lambda-1)}{\theta}\right)^{\beta}, \quad \text { and } \quad h(t)=\lambda \phi_{2}(t)
$$

then a solution $x$ of equation (1.1) either oscillates or satisfies $\lim _{t \rightarrow \infty} x(t)=0$.

Proof. Let $x(t)$ be a nonoscillatory solution of (1.1), say $x(t)>0$ for $t \in\left[t_{1}, \infty\right)_{\mathbb{T}}, x(\tau(t, \eta))>0$ for $(t, \eta) \in\left[t_{1}, \infty\right)_{\mathbb{T}} \times[a, b]$, and $x(\phi(t, \xi))>0$ for $(t, \xi) \in\left[t_{1}, \infty\right)_{\mathbb{T}} \times[c, d]$ for some $t_{1} \in$ $\left[t_{0}, \infty\right)_{\mathbb{T}}$. We need to show that $x(t) \rightarrow 0$ as $t \rightarrow \infty$.

Define the function $z$ as in (1.3). From Lemma 2.1, we can easily see that

$$
\left[r(t)\left(z^{\Delta \Delta}(t)\right)^{\alpha}\right]^{\Delta}<0 \text { and } z^{\Delta \Delta}(t)>0 \text { for } t \in\left[t_{1}, \infty\right)_{\mathbb{T}}
$$

and either $z^{\Delta}(t)>0$ or $z^{\Delta}(t)<0$ for $t \in\left[t_{2}, \infty\right)_{\mathbb{T}}$ for some $t_{2} \geq t_{1}$. 
Assume $z^{\Delta}(t)>0$ on $\left[t_{2}, \infty\right)_{\mathbb{T}}$. Then,

$$
\begin{aligned}
x(t) & =z(t)-\int_{a}^{b} p(t, \eta) x[\tau(t, \eta)] \Delta \eta \\
& \geq z(t)-\int_{a}^{b} p(t, \eta) z[\tau(t, \eta)] \Delta \eta \\
& \geq z(t)-z[\tau(t, b)] \int_{a}^{b} p(t, \eta) \Delta \eta \\
& \geq\left(1-\int_{a}^{b} p(t, \eta) \Delta \eta\right) z(t) \\
& \geq(1-P) z(t) .
\end{aligned}
$$

Using (3.4), (H4), and (H5) in (1.4), we obtain

$$
\begin{aligned}
{\left[r(t)\left(z^{\Delta \Delta}(t)\right)^{\alpha}\right]^{\Delta} } & =-\int_{c}^{d} q(t, \xi) f(x[\phi(t, \xi)]) \Delta \xi \\
& \leq-k(1-P)^{\beta} \int_{c}^{d} q(t, \xi) z^{\beta}(\phi(t, \xi)) \Delta \xi \\
& \leq-c_{1} q(t) z^{\beta}\left(\phi_{1}(t)\right) .
\end{aligned}
$$

Define the function $w(t)$ by

$$
w(t)=g(t) \frac{r(t)\left(z^{\Delta \Delta}(t)\right)^{\alpha}}{z^{\beta}(t)}
$$

Then,

$$
\begin{aligned}
w^{\Delta}(t)= & \frac{g(t)}{z^{\beta}(t)}\left(r(t)\left(z^{\Delta \Delta}(t)\right)^{\alpha}\right)^{\Delta}+\left(r(t)\left(z^{\Delta \Delta}(t)\right)^{\alpha}\right)^{\sigma}\left(\frac{g(t)}{z^{\beta}(t)}\right)^{\Delta} \\
\leq & -c_{1} g(t) q(t)\left(\frac{z\left(\phi_{1}(t)\right)}{z(t)}\right)^{\beta}+g^{\Delta}(t) \frac{\left(r(t)\left(z^{\Delta \Delta}(t)\right)^{\alpha}\right)^{\sigma}}{z^{\beta}(\sigma(t))} \\
& -g(t) \frac{\left(r(t)\left(z^{\Delta \Delta}(t)\right)^{\alpha}\right)^{\sigma}\left(z^{\beta}(t)\right)^{\Delta}}{z^{\beta}(t) z^{\beta}(\sigma(t))} .
\end{aligned}
$$

Since $r(t)\left(z^{\Delta \Delta}(t)\right)^{\alpha}$ is strictly decreasing on $\left[t_{2}, \infty\right)_{\mathbb{T}}$, we have

$$
\begin{aligned}
z^{\Delta}(t) & \geq z^{\Delta}(t)-z^{\Delta}\left(t_{2}\right) \\
& =\int_{t_{2}}^{t} \frac{\left(r(s)\left(z^{\Delta \Delta}(s)\right)^{\alpha}\right)^{1 / \alpha}}{r^{1 / \alpha}(s)} \Delta s \\
& \geq\left(r(t)\left(z^{\Delta \Delta}(t)\right)^{\alpha}\right)^{1 / \alpha} \int_{t_{2}}^{t} r^{-1 / \alpha}(s) \Delta s \\
& =R\left(t, t_{2}\right) r^{1 / \alpha}(t) z^{\Delta \Delta}(t)
\end{aligned}
$$

for $t \in\left[t_{2}, \infty\right)_{\mathbb{T}}$.

From the fact that $z(t)$ is increasing and $r(t)\left(z^{\Delta \Delta}(t)\right)^{\alpha}$ is strictly decreasing on $\left[t_{2}, \infty\right)_{\mathbb{T}}$, there exist positive constants $b$ and $b_{1}$ such that

$$
z\left(\phi_{1}(t)\right) \geq b
$$


and

$$
r(t)\left(z^{\Delta \Delta}(t)\right)^{\alpha} \leq b_{1}
$$

for $t \in\left[t_{2}, \infty\right)_{\mathbb{T}}$. Integrating the last inequality twice from $t_{2}$ to $t$, we obtain

$$
z(t) \leq z\left(t_{2}\right)+\left(t-t_{2}\right) z^{\Delta}\left(t_{2}\right)+b_{1}^{1 / \alpha} \int_{t_{2}}^{t} \int_{t_{2}}^{s} r^{-1 / \alpha}(u) \Delta u \Delta s .
$$

Thus, there exists a constant $b_{2}>0$ such that

$$
z(t) \leq b_{2} \eta^{-1}(t) \quad \text { for } t \in\left[t_{3}, \infty\right)_{\mathbb{T}}
$$

for some $t_{3} \geq t_{2}$. From (3.9) and (3.11), it is easy to see that

$$
\frac{z\left(\phi_{1}(t)\right)}{z(t)} \geq b_{3} \eta(t) \quad \text { for } t \in\left[t_{3}, \infty\right)_{\mathbb{T}}
$$

where $b_{3}:=b / b_{2}$.

Applying Keller's chain rule, we have

$$
\begin{aligned}
\left(z^{\beta}(t)\right)^{\Delta} & =\beta z^{\Delta}(t) \int_{0}^{1}\left[z(t)+h \mu(t) z^{\Delta}(t)\right]^{\beta-1} d h \\
& \geq \begin{cases}\beta\left(z^{\sigma}(t)\right)^{\beta-1} z^{\Delta}(t), & 0<\beta \leq 1, \\
\beta(z(t))^{\beta-1} z^{\Delta}(t), & \beta>1 .\end{cases}
\end{aligned}
$$

Using (3.12) and (3.13) in (3.7) implies

$$
w^{\Delta}(t) \leq-c_{1} c_{2} \eta^{\beta}(t) g(t) q(t)+g^{\Delta}(t)\left(\frac{w(t)}{g(t)}\right)^{\sigma}-\beta g(t) \frac{z^{\Delta}(t)}{z^{\sigma}(t)}\left(\frac{w(t)}{g(t)}\right)^{\sigma},
$$

for either case of $\beta$, where $c_{2}=b_{3}^{\beta}$. From (3.8) and (3.14), we then have

$$
\begin{aligned}
w^{\Delta}(t) \leq & -c_{1} c_{2} \eta^{\beta}(t) g(t) q(t)+g^{\Delta}(t)\left(\frac{w(t)}{g(t)}\right)^{\sigma} \\
& -\beta g(t) R\left(t, t_{2}\right)\left(\left(\frac{w(t)}{g(t)}\right)^{\sigma}\right)^{\frac{\alpha+1}{\alpha}}\left(z^{\sigma}(t)\right)^{\frac{\beta-\alpha}{\alpha}}
\end{aligned}
$$

for $t \in\left[t_{3}, \infty\right)_{\mathbb{T}}$.

Now, if $\beta=\alpha$, then $\left(z^{\sigma}(t)\right)^{\frac{\beta-\alpha}{\alpha}}=1$. On the other hand, if $\beta<\alpha$, then using the fact that $r(t)\left(z^{\Delta \Delta}(t)\right)^{\alpha}$ is decreasing on $\left[t_{3}, \infty\right)_{\mathbb{T}}$, we can again obtain (3.11) as above. Hence,

$$
\left(z^{\sigma}(t)\right)^{\frac{\beta-\alpha}{\alpha}} \geq c_{3}\left(\eta^{\sigma}(t)\right)^{\frac{\alpha-\beta}{\alpha}} \text { for all } t \in\left[t_{3}, \infty\right)_{\mathbb{T}}
$$

where $c_{3}=\left(b_{2}\right)^{\frac{\beta-\alpha}{\alpha}}$. Hence, for $\beta \leq \alpha$, from the definition of $\gamma(t)$ in (3.2), we have

$$
w^{\Delta}(t) \leq-c_{1} c_{2} \eta^{\beta}(t) g(t) q(t)+g^{\Delta}(t)\left(\frac{w(t)}{g(t)}\right)^{\sigma}-\beta g(t) R\left(t, t_{2}\right) \gamma(t)\left(\left(\frac{w(t)}{g(t)}\right)^{\sigma}\right)^{\frac{\alpha+1}{\alpha}}
$$

for $t \in\left[t_{3}, \infty\right)_{\mathbb{T}}$.

Taking $\lambda=\frac{\alpha+1}{\alpha}>1$,

$$
X=(\beta \gamma(t) g(t))^{1 / \lambda} R^{1 / \lambda}\left(t, t_{2}\right)\left(\frac{w(t)}{g(t)}\right)^{\sigma}
$$


and

$$
Y=\lambda^{-\alpha}\left(g^{\Delta}(t)\right)^{\alpha}\left[(\beta \gamma(t) g(t))^{-1 / \lambda} R^{-1 / \lambda}\left(t, t_{2}\right)\right]^{\alpha},
$$

we see that $X \geq 0$ and $Y \geq 0$ and so we can apply the inequality (see [12])

$$
\lambda X Y^{\lambda-1}-X^{\lambda} \leq(\lambda-1) Y^{\lambda}
$$

to obtain

$$
\beta \gamma(t) g(t) R\left(t, t_{2}\right)\left(\left(\frac{w(t)}{g(t)}\right)^{\sigma}\right)^{\frac{\alpha+1}{\alpha}}-g^{\Delta}(t)\left(\frac{w(t)}{g(t)}\right)^{\sigma} \geq-\frac{\left(\frac{\alpha}{\beta}\right)^{\alpha}\left(g^{\Delta}(t)\right)^{\alpha+1}}{(\alpha+1)^{\alpha+1}\left(\gamma(t) g(t) R\left(t, t_{2}\right)\right)^{\alpha}}
$$

for $t \in\left[t_{3}, \infty\right)_{\mathbb{T}}$. Substituting (3.17) into (3.16) gives

$$
w^{\Delta}(t) \leq-c_{1} c_{2} \eta^{\beta}(t) g(t) q(t)+\frac{\left(\frac{\alpha}{\beta}\right)^{\alpha}\left(\left(g^{\Delta}(t)\right)_{+}\right)^{\alpha+1}}{(\alpha+1)^{\alpha+1}\left(\gamma(t) g(t) R\left(t, t_{2}\right)\right)^{\alpha}} .
$$

Integrating this inequality from $t_{3}$ to $t$ yields

$$
\int_{t_{3}}^{t}\left[c_{1} c_{2} \eta^{\beta}(s) g(s) q(s)-\frac{\left(\frac{\alpha}{\beta}\right)^{\alpha}\left(\left(g^{\Delta}(s)\right)_{+}\right)^{\alpha+1}}{(\alpha+1)^{\alpha+1}\left(\gamma(s) g(s) R\left(s, t_{2}\right)\right)^{\alpha}}\right] \Delta s \leq w\left(t_{3}\right)-w(t) \leq w\left(t_{3}\right) .
$$

Taking the limsup of both sides of this inequality as $t \rightarrow \infty$, we obtain a contradiction to condition (3.1). Therefore, $z^{\Delta}(t)<0$ on $\left[t_{2}, \infty\right)_{\mathbb{T}}$.

If $x(t) \not \rightarrow 0$ as $t \rightarrow \infty$, then from Lemma 2.7, we see that (2.10) holds. Using this in equation (1.1), we obtain

$$
\begin{aligned}
{\left[r(t)\left(z^{\Delta \Delta}(t)\right)^{\alpha}\right]^{\Delta} } & =-\int_{c}^{d} q(t, \xi) f(x[\phi(t, \xi)]) \Delta \xi \\
& \leq-k \int_{c}^{d} q(t, \xi) x^{\beta}[\phi(t, \xi)] \Delta \xi \\
& \leq-k\left(\frac{1-P \theta}{\theta}\right)^{\beta} \int_{c}^{d} q(t, \xi) z^{\beta}[\phi(t, \xi)] \Delta \xi .
\end{aligned}
$$

Noting that $z(t)$ satisfies (II) in Lemma 2.1, condition (H4) implies

$$
\begin{aligned}
{\left[r(t)\left(z^{\Delta \Delta}(t)\right)^{\alpha}\right]^{\Delta} } & \leq-k\left(\frac{1-P \theta}{\theta}\right)^{\beta} z^{\beta}[\phi(t, d)] \int_{c}^{d} q(t, \xi) \Delta \xi \\
& =-k\left(\frac{1-P \theta}{\theta}\right)^{\beta} q(t) z^{\beta}\left[\phi_{2}(t)\right]
\end{aligned}
$$

for $t \in\left[t_{2}, \infty\right)_{\mathbb{T}}$. Now, for $v \geq u \geq t_{2}$,

$$
z(u)-z(v)=-\int_{u}^{v} z^{\Delta}(\tau) \Delta \tau \geq(v-u)\left(-z^{\Delta}(v)\right) .
$$

Setting $u=\phi_{2}(t)$ and $v=\lambda \phi_{2}(t)$, and using the facts that $\lambda>1$ and $\lambda \phi_{2}(t)<t$, we obtain

$$
z\left(\phi_{2}(t)\right) \geq(\lambda-1) \phi_{2}(t)\left(-z^{\Delta}\left(\lambda \phi_{2}(t)\right)\right) \quad \text { for } t \in\left[t_{2}, \infty\right)_{\mathbb{T}} .
$$

From (3.19) and (3.18), we have

$$
\left(r(t)\left(y^{\Delta}(t)\right)^{\alpha}\right)^{\Delta} \geq k\left(\frac{1-P \theta}{\theta}\right)^{\beta}(\lambda-1)^{\beta} \phi_{2}^{\beta}(t) q(t) y^{\beta}\left[\lambda \phi_{2}(t)\right]=c Q(t) y^{\beta}\left[\lambda \phi_{2}(t)\right]
$$

for $t \in\left[t_{2}, \infty\right)_{\mathbb{T}}$, where $0<y(t)=-z^{\Delta}(t)$ on $\left[t_{2}, \infty\right)_{\mathbb{T}}$. Now $y^{\Delta}(t)=-z^{\Delta \Delta}(t)<0$, so $y(t)$ is bounded. If we now proceed as in the proofs of Lemmas 2.4 and 2.5, we obtain contradictions. Hence, $x(t) \rightarrow 0$ as $t \rightarrow \infty$, and this completes the proof of the theorem. 
Remark 3.2. (i) In case

$$
\int_{t_{0}}^{t} \int_{t_{0}}^{s} r^{-1 / \alpha}(u) \Delta u \Delta s \geq t
$$

we may take

$$
\eta(t)=\left[\bar{c} \int_{t_{0}}^{t} \int_{t_{0}}^{s} r^{-1 / \alpha}(u) \Delta u \Delta s\right]^{-1} \quad \text { where } \bar{c}>0 \text { is a constant. }
$$

(ii) In case

$$
\int_{t_{0}}^{t} \int_{t_{0}}^{s} r^{-1 / \alpha}(u) \Delta u \Delta s \leq t
$$

we may take

$$
\eta(t)=\left[c^{*} t\right]^{-1} \quad \text { where } c^{*}>0 \text { is a constant. }
$$

Remark 3.3. In [16], the authors require condition (2.1) to hold and made use of Lemma 2.2 to show that in the case where $z^{\Delta}<0$, a nonoscillatory solution must converge to zero. Here we use either condition (2.3) or (2.9), and with the help of Lemma 2.7 are able to produce the same conclusion. Note also that in [16] the authors require $\alpha=\beta$ which we do not here.

Theorem 3.4. Let the hypotheses of Theorem 3.1 hold with condition (2.3) or (2.9) replaced by (2.1). Then a solution $x$ of (1.1) either oscillates or satisfies $\lim _{t \rightarrow \infty} x(t)=0$.

Proof. The proof follows from that of Theorem 3.1 and Lemma 2.2, and hence is omitted.

The following corollaries are immediate.

Corollary 3.5. Let condition (3.1) in Theorems 3.1 and 3.2 be replaced by

$$
\limsup _{t \rightarrow \infty} \int_{T}^{t} \eta^{\beta}(s) g(s) q(s) \Delta s=\infty
$$

and

$$
\limsup _{t \rightarrow \infty} \int_{T}^{t} \frac{\left(\left(g^{\Delta}(s)\right)_{+}\right)^{\alpha+1}}{\left(\gamma(s) g(s) R\left(s, T_{1}\right)\right)^{\alpha}} \Delta s<\infty
$$

for $T>T_{1} \geq t_{0}$. Then the conclusions of Theorems 3.1 and 3.2 hold.

Corollary 3.6. Assume that conditions (H1)-(H5) hold. If

$$
\limsup _{t \rightarrow \infty} \int_{T}^{t} \eta^{\beta}(s) q(s) \Delta s=\infty
$$

for $t \geq T>t_{0}$ and condition (2.3) or (2.9) holds with $Q$ and $h$ defined as in Theorem 3.1, then a solution $x$ of (1.1) either oscillates or satisfies $\lim _{t \rightarrow \infty} x(t)=0$.

Proof. This is just Theorem 3.1 with $g(t)=1$.

Next, we establish the following results.

Theorem 3.7. Let conditions (H1)-(H5) hold and assume that either condition (2.3) or (2.9) holds with $Q$ and $h$ given in (3.3). If there exists a positive non-decreasing delta differentiable function $g(t)$ such that for any positive constants $c_{2}$ and $c_{4}$ and any $T \geq t_{0}$,

$$
\limsup _{t \rightarrow \infty} \int_{T}^{t}\left[c_{1} c_{2} \eta^{\beta}(s) g(s) q(s)-\gamma_{1}(s) u^{\alpha}(s) g^{\Delta}(s)\right] \Delta s=\infty
$$


for $t \in[T, \infty)_{\mathbb{T}}$, where

$$
\gamma_{1}(t)= \begin{cases}1, & \text { if } \beta=\alpha, \\ c_{4} \eta^{\beta-\alpha}(t), & \text { if } \beta<\alpha,\end{cases}
$$

then a solution $x$ of equation (1.1) either oscillates or satisfies $\lim _{t \rightarrow \infty} x(t)=0$.

Proof. Let $x(t)$ be a nonoscillatory solution of equation (1.1), say $x(t)>0$ for $t \in\left[t_{1}, \infty\right)_{\mathbb{T}}$ for some $t_{1} \geq t_{0}$. Proceeding as in the proof of Theorem 3.1 in the case where $z^{\Delta}>0$, we again obtain inequality (3.15) which becomes

$$
\begin{aligned}
w^{\Delta}(t) & \leq-c_{1} c_{2} \eta^{\beta}(t) g(t) q(t)+g^{\Delta}(t)\left(\frac{w(t)}{g(t)}\right)^{\sigma} \\
& =-c_{1} c_{2} \eta^{\beta}(t) g(t) q(t)+g^{\Delta}(t)\left(\frac{r(t)\left(z^{\Delta \Delta}(t)\right)^{\alpha}}{z^{\beta}(t)}\right)^{\sigma} \\
& \leq-c_{1} c_{2} \eta^{\beta}(t) g(t) q(t)+r(t) g^{\Delta}(t)\left(\frac{z^{\Delta \Delta}(t)}{z(t)}\right)^{\alpha} z^{\alpha-\beta}(t)
\end{aligned}
$$

for $t \in\left[t_{3}, \infty\right)_{\mathbb{T}}$. Integrating inequality (3.8) from $t_{3}$ to $t$, we see that there exists a $t_{4} \geq t_{3}$ such that

$$
z(t) \geq\left(\int_{t_{3}}^{t} R\left(s, t_{2}\right) \Delta s\right) r^{1 / \alpha}(t) z^{\Delta \Delta}(t) \text { for } t \in\left[t_{4}, \infty\right)_{\mathbb{T}},
$$

or

$$
\left(\frac{z^{\Delta \Delta}(t)}{z(t)}\right)^{\alpha} \leq \frac{(u(t))^{\alpha}}{r(t)} \quad \text { for } t \in\left[t_{4}, \infty\right)_{\mathbb{T}} .
$$

Then (3.27) and (3.25) yield

$$
w^{\Delta}(t) \leq-c_{1} c_{2} \eta^{\beta}(t) g(t) q(t)+g^{\Delta}(t) u^{\alpha}(t) z^{\alpha-\beta}(t)
$$

for $t \in\left[t_{4}, \infty\right)_{\mathbb{T}}$.

If $\beta=\alpha, z^{\alpha-\beta}(t)=1$. If $\beta<\alpha$, from (3.11), we have

$$
z^{\alpha-\beta}(t) \leq c_{4} \eta^{\beta-\alpha}(t) \quad \text { for } t \in\left[t_{4}, \infty\right)_{\mathbb{T}},
$$

where $c_{4}=b_{2}^{\alpha-\beta}$. Using (3.29) in (3.28), we obtain

$$
w^{\Delta}(t) \leq-c_{1} c_{2} \eta^{\beta}(t) g(t) q(t)+\gamma_{1}(t) g^{\Delta}(t) u^{\alpha}(t) \text { for } t \in\left[t_{4}, \infty\right)_{\mathbb{T}} .
$$

The remainder of the proof is similar to that of Theorem 3.1 and we omit the details.

Theorem 3.8. Let conditions (H1)-(H5) hold and assume that condition (2.3) or (2.9) holds with $Q$ and $h$ given in (3.3). If

$$
\limsup _{t \rightarrow \infty} \int_{\phi_{1}(t)}^{t} q(s) u^{-\beta}\left(\phi_{1}(s)\right) \Delta s>\frac{1}{c_{1}}, \text { for } \beta=\alpha,
$$

and

$$
\lim _{t \rightarrow \infty} \int_{\phi_{1}(t)}^{t} q(s) u^{-\beta}\left(\phi_{1}(s)\right) \Delta s=\infty, \quad \text { for } \beta<\alpha,
$$

then a solution $x$ of equation (1.1) either oscillates or satisfies $\lim _{t \rightarrow \infty} x(t)=0$. 
Proof. Let $x(t)$ be a nonoscillatory solution of (1.1), say $x(t)>0$ for $t \in\left[t_{1}, \infty\right)_{\mathbb{T}}$. Proceed as in the proof of Theorems 3.1 and 3.7 to obtain inequalities (3.5) and (3.26).

Using (3.26) in (3.5) gives

$$
y^{\Delta}(t)+c_{1} q(t) u^{-\beta}\left(\phi_{1}(t)\right) y^{\beta / \alpha}\left(\phi_{1}(t)\right) \leq 0 \text { for } t \in\left[t_{3}, \infty\right)_{\mathbb{T}},
$$

where $y(t)=r(t)\left(z^{\Delta \Delta}(t)\right)^{\alpha}$. Integrating (3.32) from $\phi_{1}(t) \geq t_{3}$ to $t$ easily gives

$$
\int_{\phi_{1}(t)}^{t} q(s) u^{-\beta}\left(\phi_{1}(s)\right) \Delta s \leq \frac{1}{c_{1}} y^{1-\beta / \alpha}\left(\phi_{1}(t)\right) .
$$

Now, if $\beta=\alpha$, taking limsup as $t \rightarrow \infty$, we get a contradiction to condition (3.30); if $\beta<\alpha$, taking lim as $t \rightarrow \infty$, we obtain a contradiction to condition (3.31) due to the fact that $y(t)$ is a decreasing function. This completes the proof of the theorem.

We conclude this paper by pointing out the fact that our results are new even for the case $\mathbb{T}=\mathbb{R}$, i.e., the continuous case, and $\mathbb{T}=\mathbb{Z}$, i.e., the discrete case.

Finally, we give an example to illustrate our results.

Example 3.9. The dynamic equation

$$
\left(x(t)+\int_{2}^{3} \frac{1}{t^{2}} x\left(\eta+\frac{t}{3}\right) \Delta \eta\right)^{\Delta \Delta \Delta}+\int_{1}^{2} \frac{2 t^{2}}{\xi \sigma(\xi)} x\left(\xi+\frac{t}{4}\right) \Delta \xi=0, \quad t \in[6, \infty)_{\mathbb{T}},
$$

is a special case of (1.1) with $r(t)=1, a=2, b=3, p(t, \eta)=\frac{1}{t^{2}}, \tau(t, \eta)=\eta+\frac{t}{3}, \alpha=\beta=1$, $c=1, d=2, q(t, \xi)=\frac{2 t^{2}}{\xi \sigma(\xi)}, f(x)=x$, and $\phi(t, \xi)=\xi+\frac{t}{4}$.

Since

$$
\int_{t_{0}}^{\infty}\left(\frac{1}{r(t)}\right)^{1 / \alpha} \Delta t=\int_{6}^{\infty} \Delta t=\infty \quad \text { and } \int_{a}^{b} p(t, \eta) \Delta \eta=\int_{2}^{3} \frac{1}{t^{2}} \Delta \eta<1,
$$

conditions (H1) and (H2) hold. It is also clear that (H3)-(H5) hold.

We see that

$\int_{6}^{t} \int_{6}^{u} r^{-1 / \alpha}(s) \Delta s \Delta u=\int_{6}^{t} \int_{6}^{u} \Delta s \Delta u=\int_{6}^{t}(u-6) \Delta u \leq \int_{6}^{t} u \Delta u \leq \int_{6}^{t}(u+\sigma(u)) \Delta u=t^{2}-36 \leq t^{2}$, so for positive constants $\theta_{1}$ and $\theta_{2}$,

$$
\theta_{1} t+\theta_{2} \int_{6}^{t} \int_{6}^{u} r^{-1 / \alpha}(s) \Delta s \Delta u \leq \theta_{1} t+\theta_{2} t^{2}
$$

Hence,

$$
\eta(t)=\left[\theta_{1} t+\theta_{2} \int_{6}^{t} \int_{6}^{u} r^{-1 / \alpha}(s) \Delta s \Delta u\right]^{-1} \geq \frac{1}{\theta_{1} t+\theta_{2} t^{2}} \geq \frac{1}{\left(\theta_{1}+\theta_{2}\right) t^{2}} .
$$

We also have

$$
R\left(t, t_{1}\right)=t-t_{1} \quad \text { and } \quad q(t)=\int_{c}^{d} q(t, \xi) \Delta \xi=\int_{1}^{2} \frac{2 t^{2}}{\xi \sigma(\xi)} \Delta \xi=2 t^{2} \int_{1}^{2}\left(-\frac{1}{\xi}\right)^{\Delta} \Delta \xi=t^{2} .
$$

Condition (3.1) with $g(s)=1$ and $T>T_{1} \geq 6$ becomes

$$
\begin{gathered}
\limsup _{t \rightarrow \infty} \int_{T}^{t}\left[c_{1} c_{2} \eta^{\beta}(s) g(s) q(s)-\frac{(\alpha / \beta)^{\alpha}}{(\alpha+1)^{\alpha+1}} \frac{\left(\left(g^{\Delta}(s)\right)_{+}\right)^{\alpha+1}}{\left(\gamma(s) g(s) R\left(s, T_{1}\right)\right)^{\alpha}}\right] \Delta s \\
\quad \geq \limsup _{t \rightarrow \infty} \int_{T}^{t} c_{1} c_{2} \frac{1}{\left(\theta_{1}+\theta_{2}\right) s^{2}} s^{2} \Delta s=\limsup _{t \rightarrow \infty} \int_{T}^{t} \frac{c_{1} c_{2}}{\left(\theta_{1}+\theta_{2}\right)} \Delta s=\infty .
\end{gathered}
$$


Finally we show that (2.3) holds. Let $\lambda=\frac{4}{3}$. Since $h(t)=\lambda \phi_{2}(t)=\frac{4}{3}\left(2+\frac{t}{4}\right) \leq t$ on $[6, \infty)_{\mathbb{T}}$, and $Q(t)=\phi_{2}^{\beta} q(t)=\phi(t, d) q(t)=\left(2+\frac{t}{4}\right) q(t)=\left(2+\frac{t}{4}\right) t^{2}$, we have

$$
\begin{aligned}
\int_{h(t)}^{t} Q(s)\left(\int_{h(s)}^{h(t)} r^{-1 / \alpha}(v) \Delta v\right)^{\beta} \Delta s & =\int_{\frac{4}{3}\left(2+\frac{t}{4}\right)}^{t}\left(2+\frac{s}{4}\right) s^{2}\left(\int_{\frac{4}{3}\left(2+\frac{s}{4}\right)}^{\frac{4}{3}\left(2+\frac{t}{4}\right)} \Delta v\right) \Delta s \\
& =\int_{\frac{4}{3}\left(2+\frac{t}{4}\right)}^{t} \frac{1}{3}\left(2+\frac{s}{4}\right) s^{2}(t-s) \Delta s \\
& \geq \int_{\frac{4}{3}\left(2+\frac{t}{4}\right)}^{t} \frac{1}{3}(t-s) \Delta s \\
& \geq \frac{1}{3} \int_{\frac{4}{3}\left(2+\frac{t}{4}\right)}^{t}\left(t-\left[\frac{s+\sigma(s)}{2}\right]\right) \Delta s \\
& =\frac{1}{3} \int_{\frac{4}{3}\left(2+\frac{t}{4}\right)}^{t} t \Delta s-\frac{1}{6} \int_{\frac{4}{3}\left(2+\frac{t}{4}\right)}^{t}(s+\sigma(s)) \Delta s \\
& =\frac{1}{3} \int_{\frac{4}{3}\left(2+\frac{t}{4}\right)}^{t} t \Delta s-\frac{1}{6} \int_{\frac{4}{3}\left(2+\frac{t}{4}\right)}^{t}\left(s^{2}\right)^{\Delta} \Delta s \\
& =2\left(t^{2}-8 t+16\right) / 27 .
\end{aligned}
$$

Hence,

$$
\limsup _{t \rightarrow \infty} \int_{h(t)}^{t} Q(s)\left(\int_{h(s)}^{h(t)} r^{-1 / \alpha}(v) \Delta v\right)^{\beta} \Delta s=\infty,
$$

so the conditions of Theorem 3.1 hold, and a solution $x$ of equation (3.33) either oscillates or satisfies $\lim _{t \rightarrow \infty} x(t)=0$.

\section{References}

[1] R. P. Agarwal, M. Bohner, D. O'Regan, A. Peterson, Dynamic equations on time scales: a survey, J. Comput. Appl. Math. 141(2002), 1-26. MR1908825; url

[2] M. Bohner, A. Peterson, Dynamic equations on time scales. An introduction with applications, Birkhäuser, Boston, 2001. MR1843232; url

[3] M. Bohner, A. Peterson, Advances in dynamic equations on time scales, Birkhäuser, Boston, 2003. MR1962542; url

[4] T. CANDAN, Oscillation of second order nonlinear neutral dynamic equations on time scales with distributed deviating arguments, Comput. Math. Appl. 62(2011), 4118-4125. MR2859967; url

[5] T. Candan, Oscillatory behavior of second order nonlinear neutral differential equations with distributed deviating arguments, Appl. Math. Comput. 262(2015), 199-203. MR3346536; url

[6] D. Chen, J. Liu, Oscillation theorems for second-order nonlinear neutral dynamic equations on time scales with distributed delay (in Chinese), J. Systems Sci. Math. Sci. 30(2010), 1191-1205. MR2785243

[7] L. Erbe, T. S. Hassan, A. Peterson, Oscillation of third order nonlinear functional dynamic equations on time scales, Diff. Equ. Dyn. Syst. 18(2010), 199-227. MR2670080; url 
[8] L. Erbe, A. Peterson, S. H. Saker, Hill and Nehari type criteria for third-order dynamic equations, J. Math. Anal. Appl. 329(2007), 112-131. MR2306793; url

[9] S. R. Grace, J. R. Graef, M. A. El-Beltagy, On the oscillation of third order neutral delay dynamic equations on time scales, Comput. Math. Appl. 63(2012), 775-782. MR2880369; url

[10] J. R. Graef S. H. SAKer, Oscillation of third order nonlinear neutral functional dynamic equations, Dynam. Systems Appl. 21(2012), 583-606. MR3026094

[11] Z. HAN, T. LI, S. Sun, C. ZHANG, Oscillation behavior of third-order neutral EmdenFowler delay dynamic equations on time scales, Adv. Differ. Equ. 2010, Art. ID 586312, 23 pp. MR2588200; url

[12] G. H. Hardy, J. E. Littlewood, G. Pólya, Inequalities, reprint of the 1952 edition, Cambridge Mathematical Library, Cambridge University Press, Cambridge, 1988. MR944909

[13] T. S. Hassan, S. R. Grace, Oscillation criteria for third-order neutral nonlinear dynamic equations with distributed deviating arguments on time scales, Tatra Mt. Math. Publ. 61(2014), 141-161. MR3385234

[14] S. Hilger, Analysis on measure chains a unified approach to continuous and discrete calculus, Results Math. 18(1990), 18-56. MR1066641; url

[15] T. M. ŞENEL, Behavior of solutions of a third-order dynamic equation on time scale, J. Inequal. Appl. 2013, 2013:47, 7 pp. MR3028678; url

[16] T. M. ŞENEL, N. UTKU, Oscillation criteria for third-order neutral dynamic equations with continuously distributed delay, Adv. Difference Equ. 2014, 2014:220, 15 pp. MR3359141; url

[17] T. M. ŞENEL, N. UTKU, Oscillation behavior of third-order nonlinear neutral dynamic equations on time scales with distributed deviating arguments, Filomat 28(2014), 12111223. MR3360094; url 